\title{
Competencias docentes de los profesores de la asignatura Estudios Contemporáneos
}

Teaching competencies of the professors of the subject Contemporary Studies

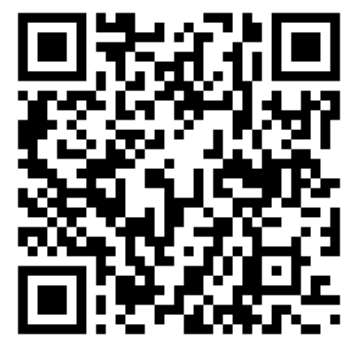

Sonnia Elizabeth Izurieta Montesdeoca ${ }^{* *}$

\section{Resumen}

Saber transferir la materia y ser especialistas en una asignatura no es suficiente en la universidad del siglo XXI pues un profesor ejerce una función de ejemplificación testimonial, desarrolla un comportamiento que sirva de modelo para los estudiantes, de ahí la importancia de construir las buenas prácticas intelectuales y de actuación. Este trabajo se planteó como objetivo, Identificar las competencias que deben poseer los docentes que dictan la materia de Estudios Contemporáneos de la Universidad Católica de Santiago de Guayaquil. Esta investigación ha sido concebida bajo el paradigma interpretativo, para lo cual se encuestó a los estudiantes, se realizó entrevistas semiestructuradas a los docentes que imparten la asignatura y se entrevistó a las autoridades que dirigen esta área humanística. Como resultado se encontró que entre las principales competencias están: ser un facilitador, apertura mental, dominio de la materia, uso de las TICs, elaboración de materiales y actualización permanentemente. Este trabajo plantea inquietudes como el perfil ideal de este docente, y las metodologías que podrían utilizar en su práctica.

\footnotetext{
* Artículo original derivado del Proyecto "Competencias docentes universitarios" Financiado por la Universidad Católica de Guayaquil entre agosto 2018 y mayo 2019

** Docente Universitaria. Universidad Católica Santiago de Guayaquil. Guayaquil, Ecuador. E-mail: sonnia.izurieta@ucsg.edu.ec._ORCID: http://orcid.org/0000-0003-3876-4113.
}

\author{
Sinergias educativas \\ Enero - junio Vol. 5 -1 - 2020 \\ http://sinergiaseducativas.mx/index.php/revista/ \\ eISSN: 2662-6661 \\ revistasinergia@soyuo.mx \\ Recepción: 17 de junio 2018 \\ Aprobación: 18 de septiembre 2019 \\ Pag 115 - 134
}

\section{Disponible en}

http://www.redalyc.org/articulo.oa?id=57356143200 9

Atribución/Reconocimiento-NoComercialCompartirlgual 4.0 Licencia Pública Internacional CC BY-NC-SA 4.0

https://creativecommons.org/licenses/by-ncsa/4.0/legalcode.es 
Palabras clave: competencias, profesorado, Estudios Contemporáneos.

\begin{abstract}
Knowing how to transfer the subject and being specialists in a subject is not enough in the university of the 21 st century because a professor exercises a role of testimonial exemplification, develops a behavior that serves as a model for students, hence the importance of building good intellectual practices and acting. This work was set as an objective, Identify the competences that teachers who have the subject of Contemporary Studies of the Catholic University of Santiago de Guayaquil. This research has been conceived under the interpretive paradigm, for which the students were surveyed, semistructured interviews were conducted with the teachers who teach the subject and the authorities that direct this humanistic area were interviewed. As a result, it was found that among the main competencies are: being a facilitator, mental openness, mastery of the subject, use of ICTs, materials development and updating permanently. This work raises concerns such as the ideal profile of this teacher, and the methodologies they could use in their practice.
\end{abstract}

Key words: competencies, teaching staff, Contemporary Studies.

\title{
Introducción
}

Muchos han sido los esfuerzos para describir las actitudes del profesor eficaz o identificar las destrezas del profesor competente, pues al tener claras estas características se podría establecer programas de formación para conseguirlas. Gimeno Sacristán y Pérez Gómez (1983) señalan que los intentos por definir las conductas del profesor han proliferado dentro del movimiento conocido como Formación del Profesorado Basada en su Competencia o Educación del Profesorado Basada en su Ejecución que tienen las características tales como que la especificación de competencias (objetivos o ejecuciones esperadas) sean observables $\mathrm{y}$ medibles, en función del progreso autónomo y autorregulado basado en las experiencias de la realidad. Estos autores mencionan que alguna de las características que debería poseer un docente son: que esté bien preparado en su asignatura, que posea una amplia 
educación general, que gobierne eficazmente la clase con técnicas efectivas de instrucción, que comprenda el proceso de aprendizaje y que mantenga un concepto adecuado de sí.

Y es que educar es algo más que informarse pues se prepara para ser ciudadanos es entonces en donde el profesor actúa como un profesional comprometido con el conocimiento para ello requiere investigar, experimentar pues debe diseñar y construir estrategias flexibles adaptadas a cada situación (Peréz Gómez, 1994 en Santos Guerra, 2001).

Si el profesor debe facilitar la construcción de ideas, modelos mentales y teorías contrastadas que le permitan buscar, seleccionar y utilizar el inagotable volumen de datos para interpretar e intervenir de la mejor manera posible en la realidad (Espacio Europeo de Educación Superior, 2009 p.5), el docente resalta la primacía de la actividad del aprendiz que incluya la implicación activa de los aprendices en el proceso de aprendizaje, refuerza la autoestima y ofrece confianza, en donde debe primar la comunicación y fomentar el uso de las Tics.

Si la universidad actual ha cambiado su rol de solo ser una ilustración de los hombres para competir con otros entes en la formación cultural para acceder al campo laboral y por otro lado resolver los dilemas de la humanidad en diferentes campos del saber, en donde el manejo y procesamiento del conocimiento se convierte en instrumento de poder en la sociedad de la información, caracterizada por un stock de saber (Suárez, 2007), es ahí donde debe formar a los sujetos, llevando a procesos de formación continua en el mundo contemporáneo. ¿Quién realizará estos procesos de cambio? Principalmente el profesor universitario, pues el formador de futuros profesionales, quien tiene que desarrollar competencias específicas para su función laboral.

¿Cuáles serían las competencias que requiere un docente en el área de un Programa Humanístico de una universidad? Para el caso de la Universidad Católica, en el año 2005 se creó la Facultad de Artes y Humanidades, una unidad académica que vincula carreras y programas de formación y que está conformada por cuatro subsistemas: Artes, Humanidades, Lenguas Extranjeras y 
Tecnologías. En los Programas de formación Humanística, se incluyen las asignaturas de Idioma Español, Introducción al Pensamiento Crítico y Estudios Contemporáneos. Los alumnos de diferentes carreras se matriculan en cada materia en los paralelos más convenientes que se distribuyen en bandas. Esta situación provocó algunas dificultades en el proceso de enseñanzaaprendizaje, pues al principio estas materias se impartían en cada Facultad pero con la creación del Programa de Humanidades, se matriculan alumnos de diferentes carreras lo cual supone una complejidad para conseguir los resultados de aprendizaje, debido en primer lugar a los intereses y conocimientos que cada estudiante posee en función de su especialidad, además el aprendizaje de ellos es más visual, no está acostumbrado a clases magistrales, a leer textos, a esta situación hay que añadir la diversidad de especialización de los profesores lo cual ocasiona que estos den mayor prioridad o énfasis en algunos contenidos de la materia, así como en la forma de impartir su clase. Esto deriva en la necesidad de hacer estudios sobre la forma como el profesorado de esta asignatura desarrolla sus clases, dado que esta asignatura forma parte de la formación humanística de los estudiantes de la Universidad Católica, es importante conocer las competencias que los docentes ponen en juego en su práctica para lograr los objetivos planteados en la materia pues la calidad del profesorado seguirá siendo una variable fundamental de la educación de calidad, lo cual debería tener reflejo en las políticas de formación, actualización, selección y de evaluación de profesores (Gimeno, 1998, p.61).

En esta investigación se plantearon las siguientes interrogantes: ¿Cuáles son las competencias que debe tener el docente de la asignatura Estudios Contemporáneos? ¿Cuáles son las competencias que utiliza el docente? ¿Cuáles son las estrategias que utiliza el docente en la cátedra de Estudios Contemporáneos? ¿Cuál es la opinión que tienen los estudiantes de las competencias que aplica el docente de Estudios Contemporáneos? ¿Qué materiales didácticos utiliza el docente para la aplicación de sus competencias?

Como objetivo general se plantea: Identificar las competencias que deben poseer los docentes que dictan la materia de Estudios Contemporáneos de la Universidad Católica de Santiago de 
Guayaquil. Este objetivo busca demostrar la hipótesis: "Los profesores que imparten la materia de Estudios Contemporáneos requieren de competencias específicas para conseguir los objetivos planteados en esta asignatura".

\section{Acercamiento al término de competencias}

Zabalza (2011) manifiesta que una competencia es un constructo molar que sirve para referirnos al conjunto de conocimientos y habilidades que los sujetos necesitan para desarrollar algún tipo de actividad. Para ello se requiere de una serie de competencias, lo que lleva a que cada competencia esté formada o diversas unidades de competencia. La Rial citado por Zabalza (2011) manifiesta que es la capacidad de usar el conocimiento y las destrezas relacionadas con productos y procesos y por consiguiente de actuar eficazmente para alcanzar un objetivo.

Para Zabalza (2003), es el conjunto de saberes éticos, vivenciales, cognitivos, emotivos y prácticos, entre los que figuran las capacidades individuales, los conocimientos, el saber hacer, las habilidades, experiencias, experiencias prácticas, actitudes y aptitudes necesarias, para que el profesional pueda desempeñar roles de trabajo específicos y realizar actividades que le conduzcan al logro de objetivos determinados.

Según Le Boterf (2001) ser competente significa "saber actuar y reaccionar con pertinencia, saber combinar los recursos y movilizarlos en un contexto, saber transferir la información, saber aprender y aprender a aprender y saber comprometerse" (p. 121). Para Gimeno Sacristán (2008) "la competencia sería la habilidad para hacer frente con éxito a las demandas complejas" (p.183).

Para Tejada (2005) "una competencia representa un conjunto de conocimientos, procedimientos y actitudes combinados, coordinados e integrados, en el sentido que el individuo ha de saber hacer y saber estar para el ejercicio profesional", (p.7). 


\section{Competencias relacionadas con la función docente}

Torres, Badillo, Valentin, \& Ramírez (2014) señalan que las competencias docentes son importantes porque:

a. Permiten desarrollar los conocimientos, habilidades y actitudes para que el alumno se desempeñe en los diferentes ámbitos de la vida social.

b. Constituyen un factor clave para formar a ciudadanos capaces de convivir en sociedades marcadas por la diversidad y prepararlos para la integración y la solidaridad.

c. Son los actores principales en el proceso de mejoramiento de la calidad educativa que promueve el desarrollo de los valores y actitudes que constituyen el saber, el saber hacer y el saber ser.

d. Representan un elemento del proceso de aprendizaje que responde a los requerimientos del proceso productivo, a las formas de organización laboral, a las nuevas tecnologías de la información y a la actualización permanente e innovadora de toda profesión (p.130).

Tejada (2013) menciona las competencias que la Comisión Europea (2008) señala:

a) Competencias profesionales, relacionadas con la experiencia laboral y las destrezas técnicas profesionales; tienen que ver con las competencias específicas de su especialidad.

b) Competencias pedagógicas y sociales, que facilitan los procesos didácticos, el trabajo con los jóvenes, la integración de las funciones formativas, la mentorización, el aprendizaje colaborativo y la trasferencia efectiva de conocimiento.

c) Competencias de gestión, coordinación con agencias formativas colaboradoras y supervisión de prácticas.

Perrenoud (2004:15-16), proporciona un decálogo de competencias de los docentes de primaria tales como:

- Organizar y animar situaciones de aprendizaje 
- Gestionar la progresión de los aprendizajes

- Elaborar y hacer evolucionar dispositivos de diferenciación

- Implicar a los alumnos en su aprendizaje y en su trabajo

- Trabajar en equipo

- Participar en la gestión de la escuela

- Informar e implicar a los padres

- Utilizar las nuevas tecnologías

- Afrontar los deberes y los dilemas éticos de la profesión

- Organizar la propia formación continua

Perrenoud (2004) da una figura de profesor ideal en el doble registro de la ciudadanía y de la construcción de competencias para ello el profesor debe ser una persona creíble, mediador intercultural, animador de una comunidad educativa, garante de la ley, organizador de una vida democrática, conductor cultural, intelectual. Es decir que sea un pedagogo constructivista, garantizar el sentido de los saberes, crear situaciones de aprendizaje, gestionar la heterogeneidad, regular los procesos y los caminos de la formación.

Ayala (2008 citado por Tejada, 2009) en su Modelo de competencias docentes alude a 5 competencias en consonancia con las funciones docentes, como se ilustra a continuación (p.176).

\section{Tabla 1}

Competencias y Funciones docentes. (Tejada, 2009 p. 6-7)

Funciones del docente

\begin{tabular}{|c|c|c|c|c|}
\hline $\begin{array}{l}\text { 1. Diseñador de } \\
\text { escenarios, } \\
\text { procesos y } \\
\text { experiencias de } \\
\text { aprendizaje } \\
\text { significativo. }\end{array}$ & $\begin{array}{l}\text { 2. Experto en } \\
\text { su disciplina } \\
\text { académica. }\end{array}$ & $\begin{array}{l}\text { 3. Facilitador y } \\
\text { guía de un } \\
\text { proceso de } \\
\text { aprendizaje } \\
\text { centrado en el } \\
\text { desarrollo } \\
\text { integral de sus } \\
\text { alumnos. }\end{array}$ & $\begin{array}{l}\text { 4. Evaluador } \\
\text { del proceso } \\
\text { de } \\
\text { aprendizaje. }\end{array}$ & $\begin{array}{lr}5 . & \text { Actor } \\
\text { consciente } & \text { del } \\
\text { proceso } & \text { de } \\
\text { cambio } & \text { en } \\
\text { educación y en un } \\
\text { contexto } \\
\text { intercultural. }\end{array}$ \\
\hline
\end{tabular}


Funciones del docente

\begin{tabular}{|c|c|c|c|c|}
\hline $\begin{array}{ll}\text { Organiza } & \text { el } \\
\text { proceso } & \text { de } \\
\text { aprendizaje } & \text { de } \\
\text { los alumnos. }\end{array}$ & $\begin{array}{l}\text { Domina } \\
\text { ampliamente } \\
\text { la disciplina } \\
\text { académica }\end{array}$ & $\begin{array}{l}\text { Brinda pautas } \\
\text { y guías para } \\
\text { llevar a cabo } \\
\text { las } \\
\text { actividades. }\end{array}$ & $\begin{array}{l}\text { Evalúa y } \\
\text { retroalimenta } \\
\text { el proceso de } \\
\text { desarrollo de } \\
\text { los alumnos. }\end{array}$ & $\begin{array}{lr}\text { Participa } & \text { en } \\
\text { procesos } & \text { de } \\
\text { investigación } & \text { e } \\
\text { innovación } & \\
\text { educativa. } & \\
\end{array}$ \\
\hline $\begin{array}{l}\text { Diseña } \\
\text { procesos y } \\
\text { experiencias de } \\
\text { aprendizaje en } \\
\text { contextos } \\
\text { reales. }\end{array}$ & $\begin{array}{l}\text { Participa en } \\
\text { procesos de } \\
\text { actualización } \\
\text { constante. }\end{array}$ & $\begin{array}{l}\text { Sugiere, } \\
\text { orienta y } \\
\text { cuestiona a los } \\
\text { alumnos } \\
\text { orientando el } \\
\text { proceso de } \\
\text { aprendizaje. } \\
\end{array}$ & $\begin{array}{l}\text { Diseña } \\
\text { rúbricas para } \\
\text { evaluar el } \\
\text { desarrollo de } \\
\text { habilidades, } \\
\text { actitudes y } \\
\text { valores. }\end{array}$ & $\begin{array}{l}\text { Se actualiza } \\
\text { constantemente y } \\
\text { se preocupa por } \\
\text { su mejora } \\
\text { continua como } \\
\text { docente. }\end{array}$ \\
\hline $\begin{array}{l}\text { Utiliza } \\
\text { tecnologías }\end{array}$ & $\begin{array}{l}\text { Actualiza el } \\
\text { curso en } \\
\text { función de } \\
\text { los avances } \\
\text { de su } \\
\text { disciplina y } \\
\text { de acuerdo al } \\
\text { nivel de sus } \\
\text { alumnos. }\end{array}$ & $\begin{array}{ll}\text { Facilita } & \text { los } \\
\text { recursos } & \\
\text { necesarios } & \\
\text { para } & \text { el } \\
\text { desarrollo de } \\
\text { actividades. }\end{array}$ & $\begin{array}{l}\text { Evalúa, } \\
\text { documenta y } \\
\text { mejora el } \\
\text { proceso de } \\
\text { enseñanza y } \\
\text { aprendizaje } \\
\text { en el curso. }\end{array}$ & $\begin{array}{lr}\text { Promueve } & \\
\text { intercambios } & \\
\text { académicos } & \text { con } \\
\text { alumnos } & \text { y } \\
\text { profesores } & \text { de } \\
\text { otras } & \\
\text { universidades. }\end{array}$ \\
\hline
\end{tabular}

Elaboración propia

Medina (2009, p.13) menciona que la competencia incluye "aquello que hay que aprender, aplicar y poner en práctica lo aprendido así como las actitudes, emociones y valores que subyacen al proceso de enseñar y aprender".

En definitiva como afirma López (2016) la competencia tiene un carácter dinámico pues puede ser aprendida y enseñada.

\section{Materiales y métodos}

Esta investigación ha sido concebida bajo el paradigma interpretativo que "recoge lo ideográfico, lo experiencial, lo trascendental, lo holístico y lo personal" (Martínez Mediano, 1996, p. 18). Se obtuvo información de los estudiantes, docentes y autoridades.

Para determinar el tamaño de la muestra de estudiantes se consideró la cantidad de estudiantes registrados en la materia de Estudios Contemporáneos en el semestre A de 2012, lo cual dio como resultado, 687 estudiantes. La muestra es no probabilística, es decir aquella en la que la elección de los elementos depende de las causas 
relacionadas con las características de la investigación o de quién hace la muestra. Se optó por un muestreo por cuotas, es decir se tomó grupos existentes en la población, es decir por ciclos en los que cursaban los alumnos y en función de esto se fijó el número de cada uno de ellos.

En cuanto a la población objeto de estudio de los profesores que impartían la materia de Estudios Contemporáneos en el Semestre B del 2012, la planta docente estaba conformada por 11 catedráticos. $\mathrm{Y}$ en lo concerniente a las autoridades se realizaron entrevistas a la decana y a la directora del área.

Con respecto a la recogida de datos de los estudiantes se utilizó el cuestionario, los ítems utilizados fueron cerrados y abiertos, para algunos casos se exigió una respuesta predeterminada, o bien una redacción personal por parte del encuestado. Por otra parte, algunas preguntas cerradas fueron elección única - una sola respuesta es posible-o múltiple -varias opciones son válidas al mismo tiempo-. En cuanto a las de elección única, algunas preguntas fueron del tipo 'lista de control', en las que la respuesta es siempre dicotómica-sí, no.

En esta investigación se realizó entrevistas semiestructuradas a 8 de los 11 profesores que dictaban clase en el semestre B-2012. También se realizaron entrevistas semiestructuradas a la Decana de la Facultad y a la Directora del Programa de Humanidades.

Se consideraron los siguientes ejes de información: el perfil ideal del docente metodología empleada, resultados de aprendizaje obtenidos, contenidos de mayor aceptación y finalidad de la asignatura.

\section{Resultados}

A continuación se presentan los resultados en función de las cinco variables estudiadas en función de las respuestas dadas por los estudiantes, docentes y directivos. 


\section{Competencias ideales del docente de Estudios Contemporáneos}

Se les preguntó tanto a los estudiantes, docentes y directivos cuales serían las principales competencias o habilidades que debería poseer un profesor que trabaja en esta asignatura. A continuación las respuestas que tuvieron mayor aceptación.

a) Dominio de la materia

Los estudiantes en un 43\% señalaron que la principal característica que deben poseer los profesores de Estudios Contemporáneos es que tenga un amplio conocimiento del tema. El 88\% de los encuestados cree que el docente de Estudios Contemporáneos reúne estas características.

Esta característica es compartida por los profesores como se puede apreciar en sus declaraciones:

Profesor EC-5: Que tenga una cultura amplia, criterio formado

Profesor EC-7: “Conozca la materia perfectamente.

Profesor EC-8: Primero demostrar que domina la materia.

Profesor EC- 10: Debe tener los conocimientos necesarios. Conocimientos de la cultura general, pues no podría un profesor coger un libro y transmitirlo porque los alumnos van a Internet y revisan los contenidos.

b) Facilitador

Mas (2012) manifiesta que el nuevo papel del docente debe "dejar el papel de reproductor de conocimiento e ir hacia un orientador de aprendizajes..." para permitir adquirir conocimientos pero especialmente saberlos buscar, procesar y aplicar" (p. 301). Se puede apreciar que los docentes coinciden con esta característica pues consideran importante las apreciaciones o juicios que realizan los estudiantes pues en la era actual de comunicación, la información está al alcance de todos y en ese sentido el rol del docente sería un facilitador. 
Profesor EC-1: Facilitador, los temas que se desarrollan son temas que están en Internet. No vamos a decir nada nuevo. A mí me interesa que ellos hablen, las apreciaciones. El chico interviene y se ve el interés.

Profesor EC- 6: El chico ha cambiado y usa las TICS, usa video youtube, se descarga uno, no tengo que dar el contenido, facilitador.

Profesor EC-5: El lenguaje debe ser cálido, pero sin llegar al coloquial. Conocer la realidad social, que no sea chapada a la antigua. Que tenga una cultura amplia, criterio formado.

Profesor EC-8: Primero demostrar que domina la materia. Un profesor debe ser un facilitador. No se debe limitar a la cátedra, En la cátedra da el tema debe abordar esto. El tema de las ideologías, exige una información mucha más rica, los sistemas económicos.

Directora: Un facilitador, más que un enciclopédico.

Decanato: No solo ser transmisor de conocimiento, ser formador y buscar resultados de aprendizaje.

c) Actualización permanente

Profesor EC-12: (...) "debe estar sumamente actualizado y debe manejar las tecnologías".

Profesor EC-3: (...) conocimientos de historia del país y el mundo. Dominio del aula, manejo de grupos porque son diversos. Que promueva la participación de los estudiantes, la criticidad, la argumentación y el manejo de las tecnologías.

Profesor EC-5: Cultura general. Que haya sido funcionario público, que tenga una actividad pública porque te da una visión amplia del Ecuador, te da acceso a muchas puertas que una persona común no tiene. No es un repetidor de contenidos, sino que los transmite. Va la experiencia. 
d) Dominio de las tecnologías

Como lo afirma Fernández (2003) "las nuevas tecnologías están transformando la ecología del aula y las funciones docentes, y estos cambios están induciendo una mutación sistemática en las teorías y en las prácticas didácticas" (p.4). Los docentes entrevistados confirman que dentro de las características esenciales para un profesor de esta área estaría el dominio de las nuevas tecnologías

Profesor EC-8: (...) Conocimiento sólido de la tecnología.

Profesor EC-3: (...) el manejo de las tecnologías

Directora: Utilice la tecnología, los recursos humanos.

e) Pensamiento Crítico

Las autoridades entrevistadas resaltaron como principales competencias del profesor de Estudios Contemporáneos las habilidades del Pensamiento Crítico, como la habilidad de la lectura, el análisis crítico de la realidad, tomar en consideración otros puntos de vista.

\section{Tabla 2}

Competencias del profesor de Estudios Contemporáneos según autoridades

\begin{tabular}{|c|c|}
\hline Visión Dirección & Visión Decanato \\
\hline $\begin{array}{l}\text { Capacidad lectora, · Análisis crítico } \\
\text { de la realidad. · No puede plantear } \\
\text { solo su punto de vista, una capacidad } \\
\text { más democrática y abierta. · Saber } \\
\text { relacionar y correlacionar los } \\
\text { contenidos }\end{array}$ & $\begin{array}{l}\text { - Dominar los conocimientos de lo } \\
\text { que va a hablar, sabiendo que el } \\
\text { estudiante con un click, puede } \\
\text { saber más que él. S Ser un pensador } \\
\text { crítico. C Capacidad de análisis. }\end{array}$ \\
\hline
\end{tabular}

Elaborado por el autor 


\section{Metodología empleada en la clase}

Medina (2012) habla de la competencia metodológica en donde resalta la importancia de que los profesores sepan seleccionar, secuenciar y estructurar didácticamente todos los contenidos de la asignatura que le permita garantizar la formación de profesionales actualizados y de alto nivel académico de ahí que en este estudio se indague sobre los métodos utilizados por el profesor de Estudios Contemporáneos..

Las principales formas de desarrollo de la clase son: intercambio de preguntas que motivan a la reflexión, explican los contenidos y discuten, los alumnos realizan exposición y presentan proyección de videos.

Como manifestaron anteriormente en el desarrollo de sus clases, la exposición de temas por parte de los alumnos, prevalece en su metodología. Añadieron también el desarrollo de cuestionarios.

Los profesores de Estudios contemporáneos utilizan como recursos para el dictado de la clase, los videos, las diapositivas. En cuanto al uso de la plataforma sólo 3 de los entrevistados la utilizan para foros, vídeos y tareas.

Los profesores mencionaron que su tutoría (trabajo práctico) tenía relación con investigaciones, exposiciones y debates. Entre los temas seleccionados estaban la identidad, la interculturalidad, las elecciones presidenciales y la cultura en cada profesión. 
Utilizan videos (Todos los profesores encuestados con excepción de EC-7)
Diapositivas Todos los profesores encuestados

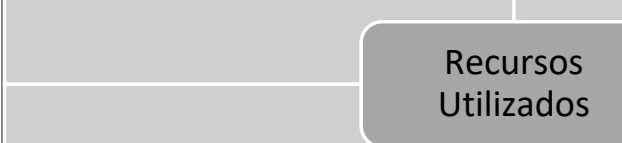

Uso de la plataforma para foros, subir videos, tareas Profesores EC-3.6, 12

No utilizan la plataforma Profesores EC-1,5,6,8,10

Figura 1 Recursos Utilizados. (Entrevistas a docentes de Estudios Contemporáneos, 2012)

Los estudiantes (50\%) manifestaron que los principales métodos utilizados en clase fueron trabajos en grupo y talleres. El otro 50\% estuvo repartido entre el uso de la plataforma moodle, la clase magistral, visitas, trabajo autónomo; el 5\% indicó que el docente utiliza otros métodos pero sin detallarlos y el 1\% no contestó. Las tareas más realizadas en esta asignatura fueron los razonamientos (19\%), las exposiciones de alumnos (18\%) y las investigaciones (17\%). En menor porcentaje se realizan otras tareas como: lecciones, debates, mapas conceptuales, etc. utilizaban diapositivas y videos principalmente. E1 89\%de los estudiantes manifestó que el profesor de Estudios Contemporáneos maneja las nuevas tecnologías y un $10 \%$ indicó que el docente no las utiliza. El principal uso que los docentes dan a las nuevas tecnologías es como recurso didáctico (50\%). El 32\% lo usa como objeto de estudio, el 10\% como medio de expresión y comunicación. Un 8\% no contestó.

\section{Resultados de aprendizajes obtenidos}

Dado que el énfasis que se da a los contenidos depende de lo que los docentes consideran como importante lograr en los estudiantes, se indagó sobre los resultados que ellos creían habían obtenido.

Al ser consultados lo docentes se observó que el primer logro el de analizar los acontecimientos históricos culturales, fue mencionado en sus intervenciones pues afirman que deben conocer sobre la realidad, que deben buscar, en cuanto a la parte de la argumentación en los logros se menciona que tenga bases, que investigue, que tenga argumentos para sus planteamientos, que tengan fundamento, el hábito de escarbar la verdad. 
Profesor EC-1: El estudiante necesita tener elevadas bases para llevar a un juzgamiento. Que los alumnos tengan una visión bastante acertada de lo que es Ecuador en el mundo de hoy.

Profesor EC-3: Fundamentalmente que puedan aplicar criterios críticos a la lectura de algunos fenómenos de la realidad, yo les pongo énfasis a eso, que sepan ser críticos, que tengan una lectura literal del texto o del video, sino que ese video le permita a él, darse cuenta que no hay una linealidad en la historia, que hay cosas que se relacionan con las otras, que si bien, hay algunos aspectos de la historia que se repiten, el contexto va cambiando y que si nosotros tenemos conciencia de que a mira esto pasó con la dictadura como lo podemos relacionar ahora.

Profesor EC-5: Si hay seriedad que el tema, los hayan puesto en atención. Crearles un hábito a la lectura o inquietud de buscar un tema que no consultan. Que lean sobre autores para que analicen como es el país.

Profesor EC-6: Conciencia de la realidad como ecuatorianos. Mayor responsabilidad de lo que pasa en el mundo y lo que se espera de hecho. Pero sobre todo que tengan argumentos para sus planteamientos, que tengan fundamento. El hábito de escarbar la verdad, que no es fácil, no todos lo tienen.

Profesor EC-7: Adquieren conocimientos y eso depende del profesor ya sea por escrito o verbal, demuestran que tienen interés.

Profesor EC-8: Que sea excelente en que conozca la realidad de la problemática del país. No lo de los textos que están desactualizados, con excepción un poco del de Ayala.

Profesor EC-12: Que tengan una idea general muy general de lo que estamos viviendo y que la puedan aplicar en hechos cotidianos y que entiendan el porqué de los diferentes sucesos, que entiendan porque Ecuador ha tenido más de presidentes de la república en los últimos años, no los que tenían que ser.

El aspecto de la cultura como un logro también fue destacado por los docentes. Este contenido abarca una unidad de la asignatura.

Profesor EC-7: No saben lo agradable que es conversar con una persona que sabe de diferentes temas en el área política y social. 
Profesor EC10: Un estándar muy alto, que ellos aprendan básicamente la parte de la cultura occidental.

Profesor EC-12: Por lo menos que manejen el término de cultura que es el más simple en realidad.

\section{Contenidos de mayor aceptación}

En función de los contenidos que se imparten los docentes emplean diversas estrategias. En muchos casos estas estrategias hacen más agradables o no algunos temas. Se indagó qué temas los alumnos consideraron importantes y a los docentes y directivos, cuál era la percepción que tenían los estudiantes sobre los tópicos de mayor interés.

Los temas que los alumnos encontraron más interesantes fueron: cultura e identidad cultural, globalización, acontecimientos mundiales del siglo XXI y del siglo XX, análisis de hechos trascendentales de la Historia del Ecuador y análisis de la deuda externa.

Los contenidos le parecieron interesantes, en un $34 \%$ porque era de su área de interés, otra razón fue porque el profesor lo explicó mejor $(25 \%)$ y porque estaba relacionado con su carrera en el $18 \%$.

Entre los temas que le parecieron no muy útiles se encontró el Auge de China, la Doctrina de la Iglesia y el análisis de la población ecuatoriana. La principal razón por la que no les parecieron muy útiles estos temas fue que no eran de su interés. Cabe recalcar que el $49 \%$ no contestó esta pregunta. Los temas que les gustaría ver son cultura actual, tecnología, política ecuatoriana e historia. Ecuatoriana.

Los temas de identidad, cultura, globalización y políticos son los que más motivan a los alumnos, entre las razones estarían que a los estudiantes les llama mucho la atención estos contenidos, porque las ideas que tenían sobre estos tópicos eran muy diferentes, y cuando los profesores de Estudios Contemporáneos los explican, contribuyen a que los discentes cambien su mentalidad y eviten los prejuicios y estereotipos. Mientras que para la Directora del área piensa que un tema motivador es el de globalización y el desarrollo de las nuevas tecnologías. 


\section{Finalidad de la asignatura}

Las creencias de los docentes condicionan sus acciones y los contenidos que enseñan. Por eso se indagó sobre la finalidad que cumple esta asignatura. Los docentes de Estudios Contemporáneos piensan que la finalidad está en ampliar la visión de lo que pasa en el mundo.

Profesor EC-5: Se trata de que un chico que se está formando en cualquier campo conozca de lo que pasa en el mundo. Como mi formación de abogado, busco por ejemplo el tema del "Buen Vivir", de los derechos. El mundo actual, el caso de abuelitos que si vino la reforma penal, vitalicia, perpetua. Ponerse en el orden del tema.

Profesor E-6:"Formar la conciencia social y ambiental del estudiante"

Profesor EC-7: "Integrar al estudiante al conocimiento de la realidad nacional, lo que le permite tener una visión clara y la responsabilidad futura de su país. Usted no puede ser responsable si no la conoce. Estoy diagnosticando para ver cuáles son las reacciones que se pueden dar en función del médico/abogado, etc.”.

Profesor EC-8: "Conocer la realidad del Ecuador, no la historia es mentirosa hay un desfase entre la realidad y la historia”.

Profesor EC-12: "Esta asignatura forma parte de las materias de humanidades, por lo tanto, tiene como objetivo que el estudiante tenga una visión más completa del mundo sobre las temáticas, que trabaja en esta materia específicamente".

Esta creencia también es compartida por las autoridades, quienes sostienen:

Directora: Analizar el pasado, el presente y de forma actual al Ecuador en los ámbitos políticos, culturales, económicos. Desde los antecedentes históricos que tenga una visión desde el mundo actual, la problemática histórica, cultural con todos los cambios. La cultura considerando todos los elementos de pueblo para el cambio de visión, humanísticas del pasado a lo que somos.

Decana: El estudiante pueda ubicarse desde el Ecuador frente a los cambios históricos y contemporáneos del mundo. 


\section{Discusión}

Se encontró que los alumnos tienen actitudes favorables hacia el profesor de Estudios Contemporáneos pues afirmaron que era cordial, respetuoso, que provoca un interés por la materia y están abiertos a nuevas ideas y la gran mayoría utilizaba los recursos tecnológicos. En lo que respecta al comportamiento del profesor es de resaltar que se considera como un facilitador, que tengan apertura mental, que domine la materia pues los jóvenes quieren más espontaneidad, calidez; debe saber utilizar las TICs, preparar materiales y actualizarse permanentemente.

Por el lado de los docentes se encontró que consideraban que la materia tenía por objetivo ampliar la visión de lo que pasa en el mundo, para ampliar o elevar el nivel cultural de los estudiantes, lo que les ayudará en su futuro ejercicio profesional. En cuanto al logro conseguido con esta asignatura está el análisis de los acontecimientos históricos culturales, enfatizando que se busca que tengan bases, argumentos para evaluarla. En lo referente a los contenidos, hay discrepancias pues unos enfatizan más el tema de la cultura y en cambio otros se van a la parte económica y de los recursos, esto probablemente debido a la fusión de las materias y a la particularidad de cara carrera.

Este estudio se ha basado en autores como Medina (2009), Pérez Gómez (2008) Perrenoud, (2004) Tejada,(2009) y Zabalza(2011). No se ha analizado las versiones de Cano (2008) y Rueda (2009).

\section{Conclusiones}

En esta investigación surgen interrogantes como: ¿Cuál sería la metodología más apropiada para abordar los contenidos de esta? ¿Qué herramientas actuales se necesita para motivar al alumno? ¿Cuáles son las nuevas tecnologías de información que demanda el estudiante actual en la sociedad del conocimiento? ¿Cómo se evaluarían esas competencias? ¿Será necesario el requerimiento de competencias mediáticas? ¿Cuáles serían las competencias digitales requeridas? ¿Cuáles son las competencias TIC? ¿Cuáles son los docentes que requiere la generación $\mathrm{Z}$ ? 


\section{Referencias}

AA, VV. (2009). Orientar el desarrollo de competencias y enseñar como aprender. La tarea del docente. Madrid: Akal

Fernández, R. (2003). Competencias profesionales del docente en la sociedad del siglo XXI. Organización y gestión Educativa: Revista del Fórum Europeo de Administradores de la Educación, 11(1), 4- 7. Recuperado de http://www.uclm.es/profesorado/ricardo/cursos/ competenciaprofesionales.pdf

Gimeno Sacristán, J \& Pérez Gómez, A. (1983). La enseñanza: su teoría y su práctica. Madrid: Akal

Gimeno Sacristán, J. (1998). Poderes inestables en educación. Madrid: Morata.

Gimeno Sacristán, J. (2008). Educar por competencias. ¿Qué hay de nuevo? Madrid: Morata

Le Boterf, G. (2001). Ingeniería de las competencias. Barcelona: Gestión 2000.

López, E. (2016). En torno al concepto de competencia: un análisis de fuentes. Profesorado. Revista de Currículum y Formación de Profesorado, 20(1), 311-322

Mas, O. (2012). Las competencias del docente universitario: la percepción del alumno, de los expertos y del propio protagonista. Revista de Docencia Universitaria, 10 (2), 299-318.

Medina, A. (2009). Fundamentación de las competencias discentes y docentes. En A. Medina. (Ed.), Formación y desarrollo de la competencias básicas (pp. 11-44). Madrid, España: Universitas.

Perrenoud, P. (2004). Diez nuevas competencias para enseñar. Barcelona: Graò. 
Santos Guierra, M. (2001). Enseñar o el oficio de aprender. Organización escolar y desarrollo profesional. España: Homo Sapiens.

Tejada, J. (2005). El trabajo por competencias en el prácticum: cómo organizarlo y cómo evaluarlo. Revista Electrónica de Investigación Educativa, 7(2), $1-\quad 31 \quad$ Recuperado de http://redie.uabc.mx/vol7no2/contents-tejada.html

Tejada, J. (2009). Competencias Docentes. Profesorado. Revista de Curriculum y formación del profesorado. 13(2). ISSN 1138-414X

Tejada, J. (2013). Profesionalización docente en la universidad: implicaciones desde la formación. Rusc, 10 (1), 170-184.

Torres, A, Badillo, M, Valentin, N \& Ramírez, E. (2014). Las competencias docentes: el desafío de la educación superior. Innovación Educativa, 14(66), 129-145.

Zabalza, M. (2003). Competencias docentes del profesorado universitario: Calidad y Desarrollo Profesional. España: Narcea.

Zabalza, M. (2011). Competencias docentes del profesorado universitario. Calidad y Desarrollo profesional. Madrid. Narcea. 